\title{
Role of the surface states in the magnetotransport properties of ultrathin bismuth films
}

\author{
N. Marcano, ${ }^{1,2,3}$ S. Sangiao, ${ }^{1,2,4}$ C. Magén, ${ }^{1,5}$ L. Morellón,,${ }^{1,2,4}$ M. R. Ibarra, ${ }^{1,2,4}$ M. Plaza, ${ }^{6}$ L. Pérez, ${ }^{7}$ and J. M. De Teresa ${ }^{1,2}$ \\ ${ }^{1}$ Departamento de Física de la Materia Condensada, Universidad de Zaragoza, 50009 Zaragoza, Spain \\ ${ }^{2}$ Instituto de Ciencia de Materiales de Aragón, CSIC-Universidad de Zaragoza, 50009 Zaragoza, Spain \\ ${ }^{3}$ Centro Universitario de la Defensa, Academia General Militar Crta. de Huesca s/n, 50090 Zaragoza, Spain \\ ${ }^{4}$ Instituto de Nanociencia de Aragón, Universidad de Zaragoza, 50018 Zaragoza, Spain \\ ${ }^{5}$ Laboratorio de Microscopías Avanzadas, Instituto de Nanociencia de Aragón-ARAID, Universidad de Zaragoza, 50018 Zaragoza, Spain \\ ${ }^{6}$ Departamento de Física de Materia Condensada, Universidad Autónoma de Madrid, 28049 Madrid, Spain \\ ${ }^{7}$ Departamento de Física de Materiales, Universidad Complutense de Madrid, 28040 Madrid, Spain \\ (Received 12 May 2010; revised manuscript received 13 July 2010; published 29 September 2010)
}

\begin{abstract}
We have investigated the magnetotransport properties of ultrathin films of Bi grown on thermally oxidized $\mathrm{Si}(001)$ substrates with thickness ranging from 10 to $100 \mathrm{~nm}$ at temperatures down to $2 \mathrm{~K}$ and in magnetic fields up to $90 \mathrm{kOe}$. Remarkable differences both in temperature and field dependence of the Hall resistivity are found for the films with thickness above and below $20 \mathrm{~nm}$. These observations can be explained due to the presence of surface states, which play an important role in determining the electronic transport properties of the thinnest films. The estimated surface carrier density $4 \times 10^{13} \mathrm{~cm}^{-2}$ at room temperature correlates well with that recently reported from angle-resolved photoemission spectroscopy on ultrathin $\mathrm{Bi}(001)$ films.
\end{abstract}

DOI: 10.1103/PhysRevB.82.125326

PACS number(s): 73.20. $-\mathrm{r}, 73.25 .+\mathrm{i}, 73.50 . \mathrm{Jt}$

\section{INTRODUCTION}

Semimetal bismuth $(\mathrm{Bi})$ is one of the most studied elements in solid-state physics because of its unique electronic properties due to its complex and highly anisotropic Fermi surface. The elongated pockets of holes and electrons with small effective masses lead to a large Fermi wavelength $\left(\lambda_{F}\right)$ of about $30 \mathrm{~nm}$, which is more than a factor of 100 larger than in regular metals. Moreover, the mean-free path in $\mathrm{Bi}$ can be as much as several millimeters, ${ }^{1}$ several orders of magnitude larger than those for most metals. These characteristics make $\mathrm{Bi}$ a suitable candidate to study both classical and quantum size effect (QSE), for which the characteristic lengths are the carrier mean-free path and Fermi wavelength, respectively. In the 1960 s, the oscillation of the film resistance with the film thickness $t$ was reported $^{2}$ and a semimetal-to-semiconductor transition caused by QSE was predicted at a critical thickness of $t \approx 30 \mathrm{~nm}^{3}$

However, after three decades of intensive research, whether such phenomena can really be observed has been argued and it is still a controversial issue. ${ }^{4-6}$ Those predictions were based on the bulk band structure but recent experiments have revealed the significant contribution from the surface band as the system downsizes to nanometer scale, making this system even more intriguing. In fact, one remarkable effect of the surface in semiconductors is the appearance of surface states with energy levels in the gap between valence and conduction bands. ${ }^{7}$ The schematic energy band diagram showing the energies of the band edges and the Fermi level for bulk $\mathrm{Bi}$ and the tentative energy band scheme for ultrathin Bi films is presented in Fig. 1. One could speculate that the $\mathrm{Bi}$ surface band could be responsible for the transport properties of $\mathrm{Bi}$ films and nanowires when the thickness is below $20-30 \mathrm{~nm}$, once the bulk bands have been pushed away from the Fermi level due to size effects. Thus, the conduction might be confined typically within one monolayer at the film surface, opening a rich physics to explore, as in the case of topological insulators. ${ }^{8}$ Thus, it seems pertinent to investigate such possibility.
To date, evidences of metallic surface states have been found in different bismuth systems. Superconductivity in granular systems built from Bi clusters where related to the strongly increased surface density of states. ${ }^{9}$ Direct observations by means of angle-resolved photoemission spectroscopy (ARPES) show that the surface states of Bi crystals are highly metallic in contrast to the semimetallic nature of bulk $\mathrm{Bi}$, with carrier densities of $\Sigma \sim 2-5 \times 10^{12} \mathrm{~cm}^{-2} \cdot{ }^{10-13}$ Hirahara et $a l .{ }^{14}$ provided evidence of large surface-state conductivity at room temperature in ultrathin $\mathrm{Bi}(001)$ films via in situ microscopic-four-point probe conductivity measurements, concluding that transport properties are dominated by these inert surface states that are Rashba spin split. ${ }^{15,16}$

So far, there have been magnetotransport studies of very thin films that showed the presence of an excess carrier density of thin films of $\mathrm{Bi}^{17,18}$ Those studies, however, were focused on thin films with thickness larger than $\lambda_{F}$. In this paper, we report on a detailed study of the magnetoresistance
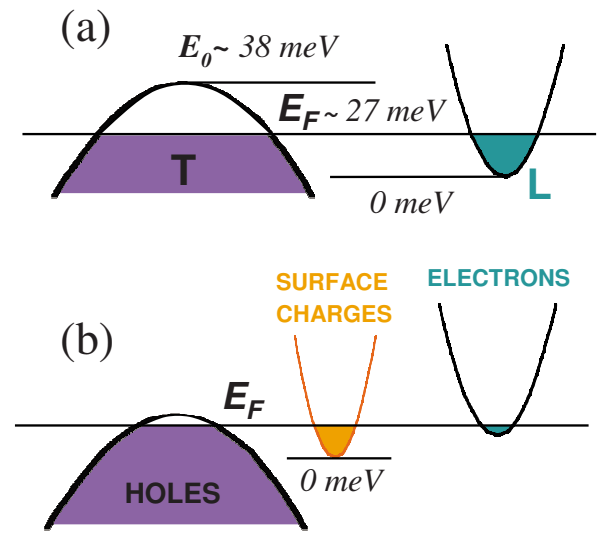

FIG. 1. (Color online) Schematic energy-band diagram showing the energies of the band edges for the L-point electron pockets and the T-point holes and the Fermi level for (a) bulk Bi, where the band overlap is $38 \mathrm{meV}$ and Fermi level is $27 \mathrm{meV}$ from the bottom of the L-point pocket band edge, and (b) tentative energy-band scheme for ultrathin Bi films (see text). 
(MR) and Hall resistivity of ultrathin Bi films grown on thermally oxidized $\mathrm{Si}(001)$ with thickness ranging from 10 to $100 \mathrm{~nm}$, for which we observe a remarkable change both in the temperature and magnetic field dependence at the thickness crossover of $20 \mathrm{~nm}$. Our results pinpoint the key role played by the surface states in the transport properties when the system downsizes below $\lambda_{F}$.

\section{EXPERIMENTAL}

The $\mathrm{Bi}$ ultrathin films, $10-100 \mathrm{~nm}$, were grown on $\mathrm{Si}(001)$ substrates by e-beam evaporation in vacuum at a pressure of $10^{-7}$ Torr at a deposition rate of $0.5 \mathrm{~nm} / \mathrm{min}$. Film thickness was controlled with a quartz crystal microbalance. Prior to the $\mathrm{Bi}$ evaporation, $200 \mathrm{~nm}$-thick $\mathrm{SiO}_{2}$ was formed on top of the Si substrate through thermal oxidation. The crystalline quality, microstructure and thickness of the samples were checked by high-resolution x-ray diffraction (XRD) and X-ray reflectivity (XRR), scanning electron microscopy (SEM) and ultrahigh-resolution transmission electron microscopy (HRTEM). XRD were performed in a D8 Advanced Bruker diffractometer and the used wavelength was that of $\mathrm{Cu} \mathrm{K} \alpha_{1}$. HRTEM experiments have been carried out in a FEI Titan Cubed TEM equipped with an image aberration corrector and operated at $300 \mathrm{kV}$. Scanning transmission electron microscopy (STEM) combined with electron energy loss spectroscopy (EELS) and energy dispersive $\mathrm{x}$-ray spectroscopy (EDX) have been performed in the FEI Titan TEM equipped with a probe corrector, a Gatan Tridiem 866 ERS energy filter and an EDAX x-ray spectrometer and operated at $300 \mathrm{kV}$. The lamella for the cross-sectional TEM study has been fabricated in a FEI Helios 600 Nanolab with the ion column operated at $5 \mathrm{kV}$. MR and Hall effect were carried out in a commercial PPMS from Quantum Design by means of conventional four-contact technique. The samples were patterned by a two-step optical lithography process in order to have a well-defined geometry for the transport measurements. ${ }^{19}$ The lateral size of the typical electrode for the flow of the current was $4 \mu \mathrm{m}$ and titanium pads were patterned for the measurements of the voltage drop. In all measurements, the magnetic field $H$ was applied perpendicular to the film plane and the current flow was within the film plane, parallel to the substrate edge. To cancel the effect of the field on the longitudinal component of the resistivity contributing to the measured voltage, we swept the field to positive values and then to negative values and defined the Hall signal as half the difference between the signals measured at positive and negative fields.

\section{RESULTS AND DISCUSSION}

Figure 2(a) shows the $\theta / 2 \theta$ x-ray diffraction pattern obtained for the $\mathrm{Bi} 100 \mathrm{~nm}$-thick films at room temperature as representative of the structural analysis. Bismuth has a rhombohedral crystal structure, which is usually described in a hexagonal system. The films used in this work are polycrystalline but strongly textured as evidenced by strong (003), (006), and (009) peaks which indicates that the $\mathrm{Bi}$ grains preferentially grow with the trigonal axis [001] perpendicular
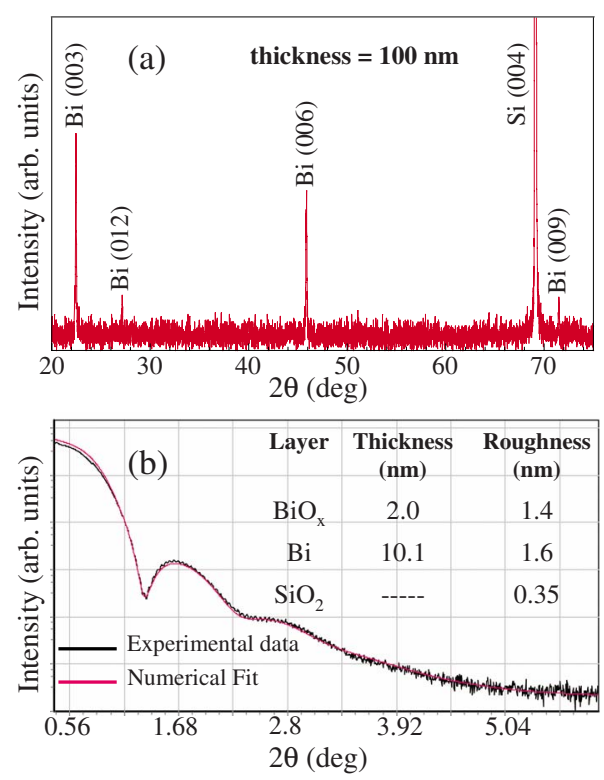

FIG. 2. (Color online) (a) X-ray diffraction pattern of the 100-nm Bi film and (b) XRR measurement from the 10-nm thick Bi film and the numerical fit described in the text. The obtained values of thickness and roughness of all layers have been listed as an inset.

to the plane of the film. SEM images reveal that the samples consist of grains with sizes comparable to the film thickness (i.e., ranging from 10 to $100 \mathrm{~nm}$ ). Film thickness has been determined by XRR measurements. Figure 2(b) shows XRR measurements for the $10 \mathrm{~nm}$-thick Bi film. The thickness and roughness of the layers have been numerically analyzed by means of the commercial software LEPTOS provided by Bruker. From the numerical analysis of the XRR data, the film thickness was found to be $10.1 \mathrm{~nm}$ and the roughness of the layer $1.6 \mathrm{~nm}$. A layer of Bi oxide (2 nm thick and the roughness of the layer $1.4 \mathrm{~nm}$ ) needs to be considered in order to reproduce the experimental data.

HRTEM images on 10-nm film, as representative of this study, reveal the crystal structure and morphology of the $\mathrm{Bi}$ thin films, as illustrated in Fig. 3. The film has polycrystalline nature textured with the (001) trigonal direction out of plane and is composed of flat nanocrystals about 10-12 nm thick and elongated along the growth plane. An example of one of these grains is displayed in Fig. 3(a) whereas Fig. 3(b) plots the Fast Fourier Transform of the area of the image where the grain is, showing that the crystal symmetry and lattice reflections match those of hexagonal $\mathrm{Bi}$ oriented along
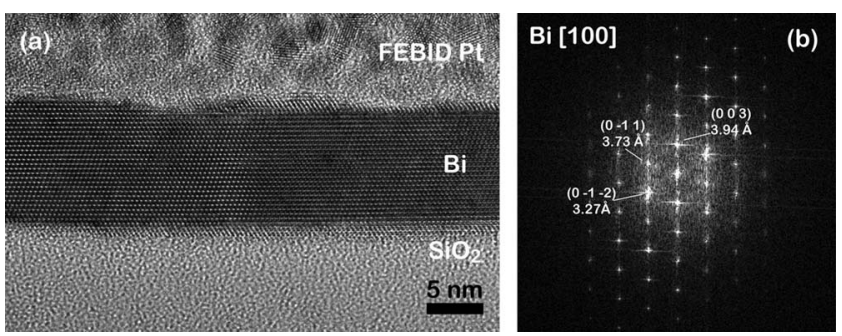

FIG. 3. (a) HRTEM image of Bi thin film grown on $\mathrm{SiO}_{2}$ layer and capped with a FEBID Pt protective layer. (b) FFT of the Bi film showing the crystal symmetry and the main lattice periodicities. 

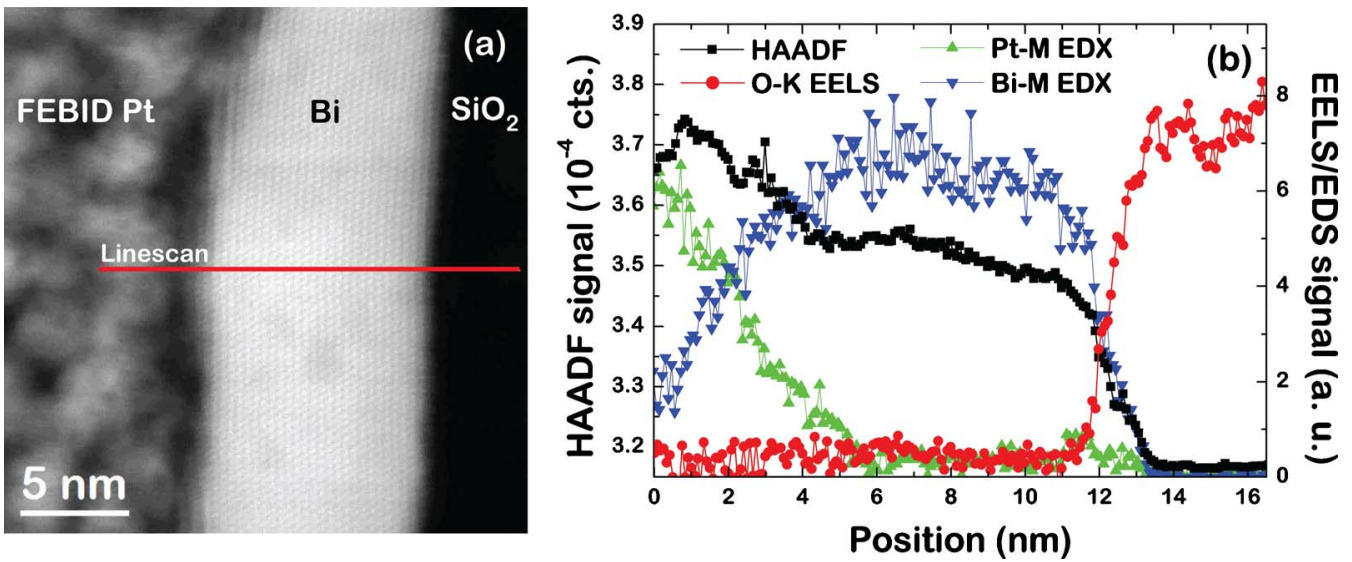

FIG. 4. (Color online) (a) HAADF STEM image of Bi thin film used as a reference image for EELS-EDX linescan. (b) Integrated EELS and EDX signals corresponding to the $\mathrm{O} K$ edge (EELS), and the $\mathrm{Bi} M$ and $\mathrm{Pt} M$ edge (EDX). HAADF signal acquired simultaneously to the spectrum profile is also represented.

the [100] axis. Relatively rough interfaces with the $\mathrm{SiO}_{2}$ buffer and with the focused electron beam induced deposit of $\mathrm{Pt}$ used as a protective layer during lamella preparations cause some contrast intermixing which makes impossible to determine the nature of the interfaces and the interface with the substrate. To clarify this point, STEM-EELS/EDX experiments have been performed to characterize the chemical composition of the surface of the films. Figure 4(a) shows the high angle annular dark field (HAADF) STEM image of one of the grains, showing features similar to those already deduced from the HRTEM experiments. Using this image as a reference, an EELS and EDX spectrum image have been collected along the line in Fig. 4(a). Figure 4(b) displays the profile of EELS oxygen integrated signal extracted from the background substracted $\mathrm{O} K$ edge, the integrated signal of EDX Pt $M$ and $\mathrm{Bi} M$ edges, and the simultaneously acquired HAADF signal. Several conclusions can be extracted from this linescan. First, the oxygen signal is concentrated in the $\mathrm{SiO}_{2}$ layer and abruptly decreases to noise level at the interface with the Bi layer. No oxygen is observed in the upper surface, the one in contact with the Pt layer, so we can conclude that $\mathrm{Bi}$ is metallic with negligible surface oxidation. In addition, $\mathrm{Bi}$ surface presents a higher roughness than the interface with the buffer, as can be noticed from the sharper $\mathrm{O}$ and $\mathrm{Bi}$ profiles of the latter interface with respect to the $\mathrm{Pt}$ and $\mathrm{Bi}$ profiles at the surface of the $\mathrm{Bi}$ film. We need to underline that the $\mathrm{Bi} M$ edge chosen to make the profile overlaps in energy with other Pt edges, so the Bi signal does not go to zero at the $\mathrm{Pt}$ protective layer whereas the Pt $M$ peak is not overlapped with other elements signal. It is intriguing that the interface of the $\mathrm{Bi}$ grain is not oxidized according to the TEM images whereas the $\mathrm{x}$-ray reflectivity measurements indicate an average $2 \mathrm{~nm}-\mathrm{BiO}_{x}$ layer in the film. One possible explanation is that some $\mathrm{Bi}$ grains are oxidized whereas other ones are not. Another explanation can be that the high-vacuum atmosphere of the TEM experiments tend to remove the oxide layer.

Figures 5(a) and 5(b) show the Hall resistivity $\left(\rho_{\text {Hall }}\right)$ isotherms from room temperature down to $2 \mathrm{~K}$ for the Bi films with thicknesses $t=10$ and $100 \mathrm{~nm}$ as representative of the present study. According to the observed temperature and field dependence the films can be divided into two groups: (i) films with $t \geq 20 \mathrm{~nm}$, where $\rho_{\text {Hall }}$ is not linear with field, changing its sign from negative at room temperature into positive below $\sim 100 \mathrm{~K}$ and (ii) films with $t<20 \mathrm{~nm}$, where $\rho_{\text {Hall }}$ displays a linear field dependence remaining its sign negative for the whole temperature range. The remarkable change both in the magnitude and the field dependency of $\rho_{\text {Hall }}$ with film thickness is illustrated in Fig. 6 , in which $\rho_{\text {Hall }}$

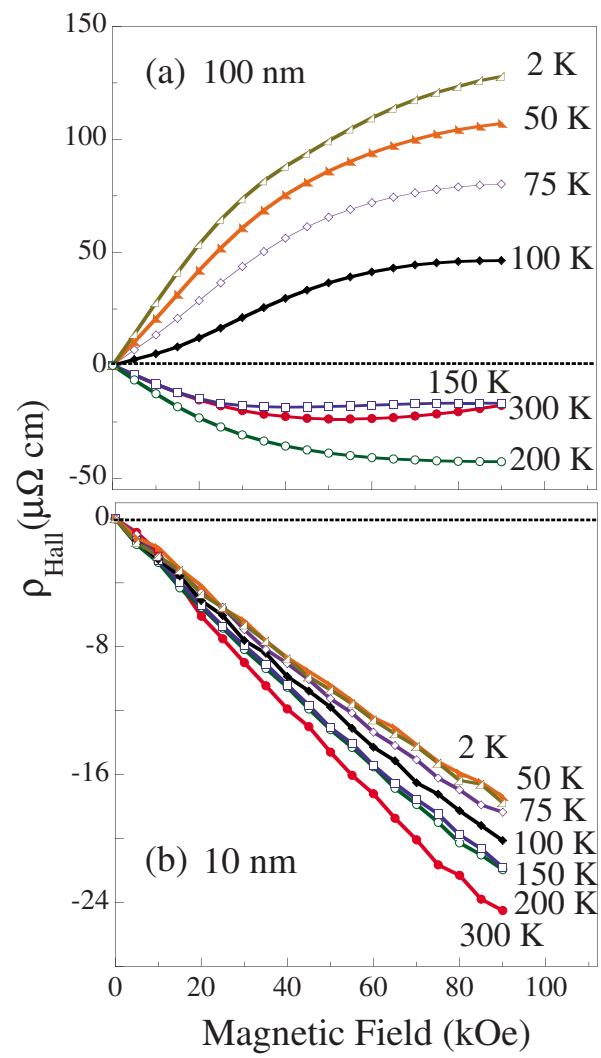

FIG. 5. (Color online) Hall resistivity $\rho_{\text {Hall }}$ isotherms at selected temperatures in the 2 to $300 \mathrm{~K}$ range for the sake of clarity as a function of magnetic field for (a) $t=100 \mathrm{~nm}$ and (b) $t$ $=10 \mathrm{~nm}$-thick Bi films as representative samples of the present study. Solid lines drawn are guide to the eyes. 


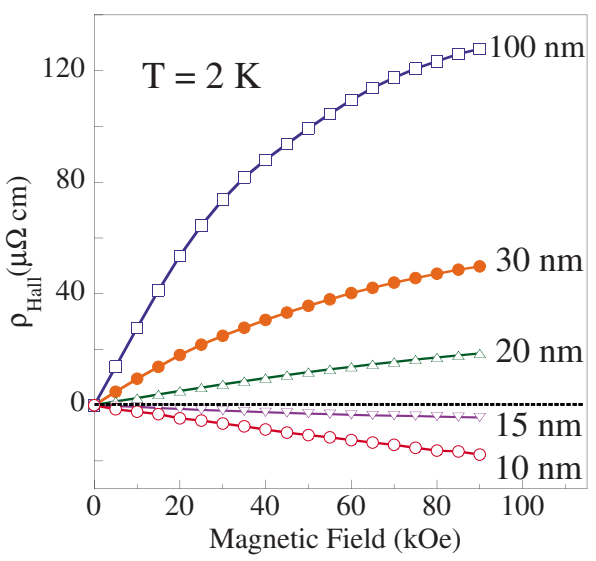

FIG. 6. (Color online) Hall resistivity $\rho_{\text {Hall }}$ as a function of magnetic field for several film thickness in the 10 to $100 \mathrm{~nm}$ range as representative of this study at $2 \mathrm{~K}$. Solid lines drawn are guide to the eyes.

as a function of magnetic field is compared for different film thickness down to $10 \mathrm{~nm}$ at $2 \mathrm{~K}$.

The observation of a linear Hall effect in the $10 \mathrm{~nm}$-thick film from 0 to $90 \mathrm{kOe}$ in the whole temperature range is particularly remarkable. One expects the surface carriers to become important or even a clear majority as film thickness is reduced due to the large surface area per unit volume. Furthermore, finite size or quantum effects in ultrathin films can shift the position of the electron and hole bulk bands, leading to even greater relevance of the surface band. Indeed, according to our analysis, the carrier concentration of the surface band $n_{s}$ increases with respect to the bulk bands as the thickness of the film is reduced.

In the following, we use the conventional theory of multiband transport ${ }^{20}$ to extract the electron and hole carrier densities and mobilities from a simultaneous fitting of the temperature and field evolution of the MR and Hall resistivity $\rho_{\text {Hall }}$. According to such a model, each band has two parameters, resistivity $\rho_{i}$ and Hall coefficient $R_{i}=1 / q_{i} n_{i}$ where $q_{i}$ $= \pm e$ is the charge of the carrier. Each band contributes to the conductivity of the system in parallel with the other bands. Thus, the total conductivity is a sum of the contributions from all the bands $\hat{\sigma}=\sum_{i=1} \hat{\sigma}_{i}$ and the total resistance is $\hat{\rho}=\hat{\sigma}^{-1}$. It should be pointed out that this model does not provide the complete description of the system since the anisotropy in the electron mobilities found in bulk Bi is not being taken into account. Such an effect can have a minor role in our Bi films as they are not single crystalline. This is why we restrict the model to the interplay of three isotropic bands, which in fact provides a good fit to the experimental data.

Figure 7 shows the magnetic field dependence of the resistance $[\rho(H)-\rho(H=0)]$ and $\rho_{\text {Hall }}$ at a selected temperature $T=50 \mathrm{~K}$ for film thickness $t=10,30$, and $100 \mathrm{~nm}$ as representative of this study. These results have been fitted to the above mentioned model. In agreement with earlier analysis of the magnetotransport properties of $300 \mathrm{~nm}$-thick $\mathrm{Bi}(001)$ films we started with a simple two-band model, with a bulk electron and a bulk hole band. ${ }^{21}$ In that case, the nonlinear magnetic field dependence of the Hall resistivity was ex-

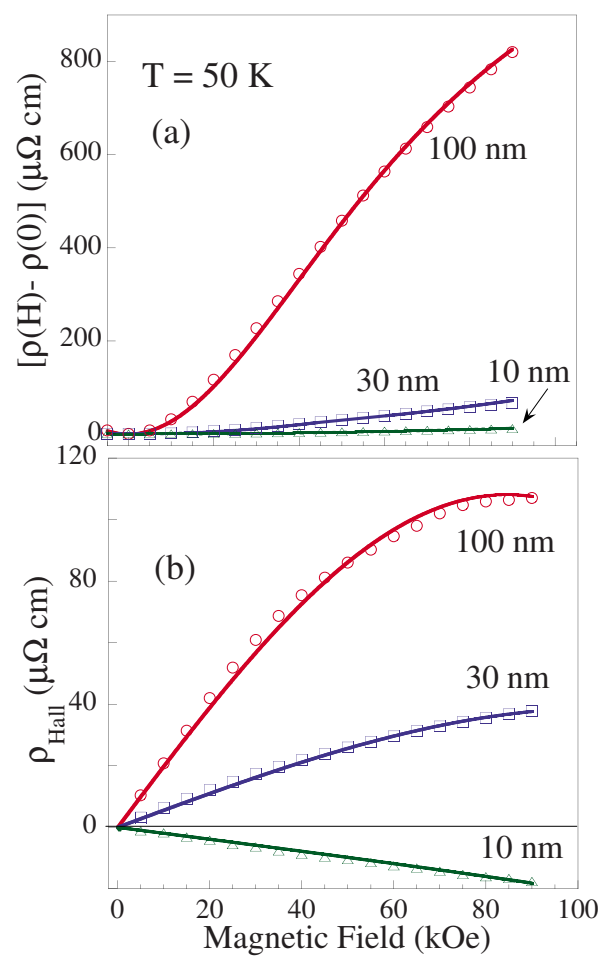

FIG. 7. (Color online) (a) Magnetoresistance (top panel) and Hall resistivity $\rho_{\text {Hall }}$ (bottom panel) versus applied field at selected temperature $T=50 \mathrm{~K}$ for Bi films with thickness $t=100,30$, and 10 $\mathrm{nm}$ as representative of this study. The solid lines are determined by a fitting procedure that simultaneously includes both sets of data into a three-band model described in the text.

plained by the noncompensated electron and hole bulk bands. This attempt is shown in Fig. 8, which displays the magnetic field dependence of the resistance and $\rho_{\text {Hall }}$ at $50 \mathrm{~K}$ only for $100 \mathrm{~nm}$-thick film for the sake of clarity. Black solid line is calculated from two band model. It is clear that, in the present study, the inclusion of a third carrier band (with $\rho_{s}$ and $R_{s}=1 / e n_{s}$ as characteristic band parameters) is required to account for the temperature and field evolution of the magnetoresistance and $\rho_{\text {Hall }}$ (red solid line). We fit MR and $\rho_{\text {Hall }}$ simultaneously by adjusting the six parameters $(n, p$, $\mathrm{R}_{n}, \mathrm{R}_{p}, \mathrm{n}_{s}$, and $\mathrm{R}_{s}$ ) independently, until differences between the fitting curves and the experimental data are minimized. The solid lines in Figs. 7(a) and 7(b) are calculated from our three-band model analysis. The experimental and calculated resistances are in very good agreement for the whole magnetic field range.

For the 100 nm-thick film we obtain $n \approx p \approx 5$ $\times 10^{18} \mathrm{~cm}^{-3}$ over $100 \mathrm{~K} \leq T \leq 300 \mathrm{~K}$ temperature range. Those values are in agreement with those reported for 100 nm-thick Bi epitaxial films. ${ }^{4,17,22}$ It is worth noting that the three-band model with electron and hole bulk bands together with a minority surface electron band have been recently reported to describe the magnetotransport properties on 100 nm-width Bi nanowires. ${ }^{23}$ In the present case, however, the third band can be interpreted as an effective band which contains surface carriers characterized by an effective density and an effective mobility. This model accounts for two possible scenarios: (i) surface electron and hole bands where 


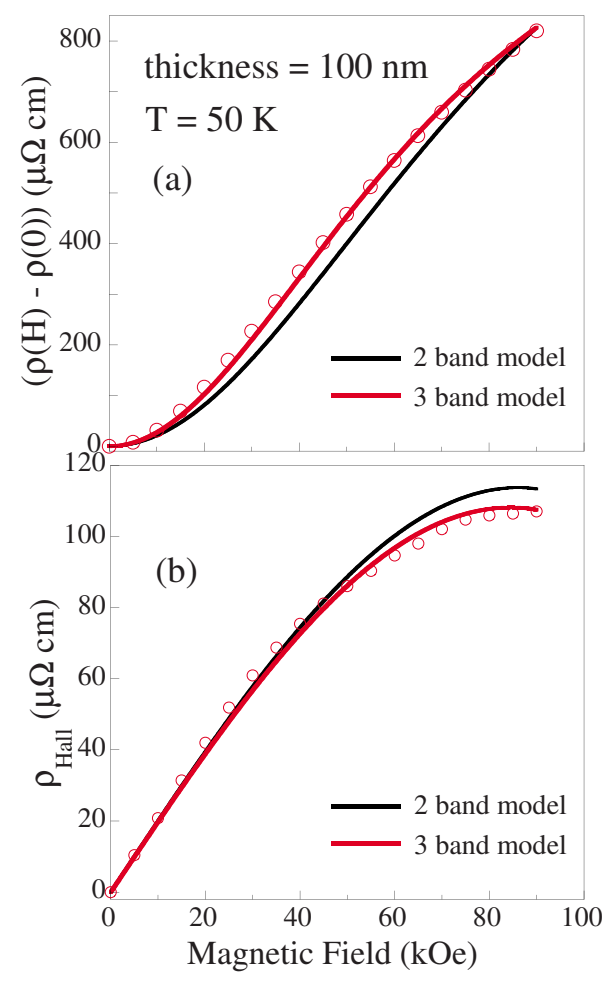

FIG. 8. (Color online) (a) Magnetoresistance (top panel) and Hall resistivity $\rho_{\text {Hall }}$ (bottom panel) versus applied field at selected temperature $T=50 \mathrm{~K}$ for Bi films with thickness $t=100 \mathrm{~nm}$. The solid lines are determined by a fitting procedure that simultaneously includes both sets of data into a two-band (black line) and a threeband model (red line) described in the text.

concentrations of electrons and holes are compensated, or (ii) dominant electron situation with higher mobility than holes. Both possibilities can account for the experimental data presented in this work. Unfortunately, the present measurements do not allow us to discern between them. The present study allows the analysis of the evolution of the ratio of $n_{s}$ to bulk electrons or holes $(n, p)$ with film thickness. For the $30 \mathrm{~nm}$ film, such a ratio is $\sim 2$ at $2 \mathrm{~K}$ whereas it increases up to $\sim 5$ at $2 \mathrm{~K}$ for the $10 \mathrm{~nm}$-thick film. For the latter, we obtain $n_{s}$ $=4 \times 10^{19} \mathrm{~cm}^{-3}$ at room temperature. The surface charge per unit area $\Sigma$ estimated as $n_{s} \times t$ gives rise to $\Sigma \sim 4$ $\times 10^{13} \mathrm{~cm}^{-2}$ in rough agreement with the $\Sigma$ determined via ARPES measurements for ultrathin $\operatorname{Bi}(001)$ films. ${ }^{14}$ The mo- bility $\mu_{i}=1 / n_{i} q_{i} R_{i}$ and the carrier density $n_{i}$ for each band obtained from fits to the three band model for the $100 \mathrm{~nm}, 30$ $\mathrm{nm}$, and $10 \mathrm{~nm}$-thick films for selected temperatures $(T$ $=5 \mathrm{~K}$ and $T=50 \mathrm{~K}$ ) are listed in Table I. For the $10 \mathrm{~nm}$ thick film the low-temperature magnetoresistance $(T \leq 5 \mathrm{~K})$ presents an extra contribution coming from the localization effects (weak antilocalization) ${ }^{24}$ which has been taken into account in the corresponding analysis.

It is worth noting that in spite of the simplicity of the multiband model, this analysis has been successful in describing the field-dependent resistivity and hall resistivity observed in this work. This approach has enable us to obtain the temperature dependence of the electron and hole densities in each sample and to identify the presence of surface carriers which play a remarkable role in determining the magnetotransport properties of the $10-\mathrm{nm}$ thick film. Such surface carriers are expected to become a clear majority and thus, dominate the electronic transport in ultrathin Bi films as thickness decreases, once the bulk bands have been shifted with respect to the Fermi level due to size effects. In fact, in situ micro-four-point-probe measurements showed that the room temperature properties of $2 \mathrm{~nm}$-thick films were dominated by the surface states and not by the states inside the film. ${ }^{14}$ Unfortunately, our attempts to grow continuous films with such low thickness were not successful.

The attribution of the third band to surface states is strongly supported by the estimated surface carrier density which is in rough agreement with the $\Sigma$ determined via ARPES in ultrathin Bi (001) films made in deposition under ultrahigh vacuum on in situ cleaned Si surfaces. ${ }^{14}$ However, the presence of an impurity band coming from the $\mathrm{Bi} / \mathrm{SiO}_{2}$ interface or the film surface $\mathrm{Bi} / \mathrm{BiO}_{x}$ can be considered as another plausible scenario which would account for the presence of extra carriers in our films. Although the STEMEELS/EDX experiments indicate the high quality of the films and the interfaces, as well as the absence of Bi oxide on the surface, further experiments beyond the scope of the present manuscript would be required to discern between both scenarios.

\section{CONCLUSIONS}

The thickness and temperature dependence of the magnetoresistance and Hall effect in ultrathin Bi films have been studied and analyzed according to the multiband model. We

TABLE I. Transport parameters for the 100, 30, and $10 \mathrm{~nm}$-thick films as representative samples of the present study at selected temperatures, $T=5 \mathrm{~K}$ and $T=50 \mathrm{~K}$, determined by the three-band model analysis described in the text.

\begin{tabular}{crccccrr}
\hline \hline $\begin{array}{c}\text { Film thickness } \\
(\mathrm{nm})\end{array}$ & $\begin{array}{c}T \\
(\mathrm{~K})\end{array}$ & $\begin{array}{c}p \\
\left(10^{18} / \mathrm{cm}^{3}\right)\end{array}$ & $\begin{array}{c}n \\
\left(10^{18} / \mathrm{cm}^{3}\right)\end{array}$ & $\begin{array}{c}n_{s} \\
\left(10^{18} / \mathrm{cm}^{3}\right)\end{array}$ & $\begin{array}{c}\mu_{p} \\
\left(\mathrm{~cm}^{2} / \mathrm{V} \mathrm{s}\right)\end{array}$ & $\begin{array}{c}\mu_{n} \\
\left(\mathrm{~cm}^{2} / \mathrm{V} \mathrm{s}\right)\end{array}$ & $\begin{array}{c}\mu_{n_{s}} \\
\left(\mathrm{~cm}^{2} / \mathrm{V} \mathrm{s}\right)\end{array}$ \\
\hline 100 & 50 & 1.74 & 4.9 & 9.4 & 677.5 & 212.7 & 139.5 \\
100 & 5 & 0.82 & 5.7 & 10.2 & 633.7 & 131.8 & 79.5 \\
30 & 50 & 7.1 & 2.8 & 8.9 & 630.1 & 576.1 & 344.2 \\
30 & 5 & 4.5 & 6.2 & 8.4 & 711.2 & 328.5 & 292.6 \\
10 & 50 & 8.12 & 13.2 & 23.6 & 134.5 & 91.4 & 57.2 \\
10 & 5 & 7.1 & 13.9 & 31.3 & 35.4 & 23.7 & 11.1 \\
\hline \hline
\end{tabular}


show that the remarkable changes observed in the magnetotransport as film thickness is reduced below Fermi wavelength $(t<30 \mathrm{~nm})$ can, within the uncertainty outlined above, be interpreted as due to the appearance of surface states, which are found to play an important role in determining the transport properties of these films. Our results indicate that both bulk and surface carriers participate in the conduction process even in the thinnest $(10 \mathrm{~nm})$ studied film but with increasing contribution of the surface band as the film thickness decreases. Our results allow one to conclude that ultrathin bismuth films can habilitate the study of electronic effects approaching two-dimensional systems, as occurs in few-layer graphene.

\section{ACKNOWLEDGMENTS}

This work was supported by Spanish Ministry of Science (through Projects No. MAT2007-65965-C02-02 and No. MAT2008-06567-C02) including FEDER funding, the Aragón Regional Government and the Universidad Complutense de Madrid. N.M. and S.S. acknowledge financial support from Spanish CSIC (JAE program) and Spanish MEC (FPU program). M.P. acknowledges Comunidad de Madrid and European Social Fund for financial support. The authors are especially thankful to I. Rivas, R. Valero, L. Casado, and R. Córdoba for assistance with optical lithography, XRD and lamellae preparation.
${ }^{1}$ R. Hartman, Phys. Rev. B 181, 1070 (1969).

${ }^{2}$ Yu. F. Ogrin, V. N. Lutskii, and M. I. Elison, JETP Lett. 3, 71 (1966).

${ }^{3}$ V. B. Sandomirskii, Sov. Phys. JETP 25, 101 (1967).

${ }^{4}$ C. A. Hoffman, J. R. Meyer, F. J. Bartoli, A. Di Venere, X. J. Yi, C. L. Hou, H. C. Wang, J. B. Ketterson, and G. K. Wong, Phys. Rev. B 48, 11431 (1993).

${ }^{5}$ H. T. Chu, Phys. Rev. B 51, 5532 (1995).

${ }^{6}$ E. I. Rogacheva, S. N. Grigorov, O. N. Nashchekina, S. Lyubchenko, and M. S. Dresselhaus, Appl. Phys. Lett. 82, 2628 (2003).

${ }^{7}$ K. Seeger, Semiconductor Physics (Springer-Verlag, Wien, 1973).

${ }^{8}$ D. Hsieh, D. Qian, L. Wray, Y. Xia, Y. S. Hor, R. J. Cava, and M. Z. Hasan, Nature (London) 452, 970 (2008).

${ }^{9}$ B. Weitzel and H. Micklitz, Phys. Rev. Lett. 66, 385 (1991).

${ }^{10}$ C. R. Ast and H. Höchst, Phys. Rev. Lett. 87, 177602 (2001).

${ }^{11}$ Yu. M. Koroteev, G. Bihlmayer, J. E. Gayone, E. V. Chulkov, S. Blugel, P. M. Echenique, and Ph. Hofmann, Phys. Rev. Lett. 93, 046403 (2004).

${ }^{12}$ T. Hirahara, T. Nagao, I. Matsuda, G. Bihlmayer, E. V. Chulkov, Yu. M. Koroteev, P. M. Echenique, M. Saito, and S. Hasegawa, Phys. Rev. Lett. 97, 146803 (2006).

${ }^{13} \mathrm{Ph}$. Hofmann, Prog. Surf. Sci. 81, 191 (2006).

${ }^{14}$ T. Hirahara, I. Matsuda, S. Yamazaki, N. Miyata, S. Hasegawa, and T. Nagao, Appl. Phys. Lett. 91, 202106 (2007).

${ }^{15}$ T. Hirahara, K. Miyamoto, I. Matsuda, T. Kadono, A. Kimura, T.
Nagao, G. Bihlmayer, E. V. Chulkov, S. Qiao, K. Shimada, H. Namatame, M. Taniguchi, and S. Hasegawa, Phys. Rev. B 76, 153305 (2007)

${ }^{16}$ T. Hirahara, K. Miyamoto, A. Kimura, Y. Niinuma, G. Bihlmayer, E. V. Chulkov, T. Nagao, I. Matsuda, S. Qiao, K. Shimada, H. Namatame, M. Taniguchi, and S. Hasegawa, New J. Phys. 10, 083038 (2008).

${ }^{17}$ D. L. Partin, J. Heremans, D. T. Morelli, C. M. Thrush, C. H. Olk, and T. A. Perry, Phys. Rev. B 38, 3818 (1988).

${ }^{18}$ C. A. Hoffman, J. R. Meyer, F. J. Bartoli, A. Di Venere, X. J. Yi, C. L. Hou, H. C. Wang, J. B. Ketterson, and G. K. Wong, Phys. Rev. B 51, 5535 (1995).

${ }^{19}$ J. M. De Teresa, A. Fernández-Pacheco, L. Morellon, J. Orna, J. A. Pardo, D. Serrate, P. A. Algarabel, and M. R. Ibarra, Microelectron. Eng. 84, 1660 (2007).

${ }^{20}$ N. W. Ashcroft and N. D. Mermin, Solid State Physics (Holt, Rinehart, and Winston, New York, 1976).

${ }^{21}$ N. Marcano, S. Sangiao, J. M. De Teresa, L. Morellón, M. R. Ibarra, M. Plaza, and L. Pérez, J. Magn. Magn. Mater. 322, 1460 (2010).

${ }^{22}$ Yu. F. Komnik and V. V. Andrievskii, Sov. J. Low Temp. Phys. 1, 51 (1975)

${ }^{23}$ N. Marcano, S. Sangiao, M. Plaza, L. Pérez, A. Fernández Pacheco, R. Córdoba, M. C. Sánchez, L. Morellón, M. R. Ibarra, and J. M. De Teresa, Appl. Phys. Lett. 96, 082110 (2010).

${ }^{24}$ S. Sangiao, N. Marcano, L. Morellón, M. R. Ibarra, and J. M. De Teresa (unpublished). 